\title{
Reducing the Risk of Zika and other Arboviruses by Rapidly Eliminating Aedes albopictus Larvae from Tires Using a Spray Formulation of Bacillus thuringiensis israelensis.
}

\author{
LTC (ret) Dr. Thomas M. Kollars, Jr. \\ College of Health Sciences, Liberty University, Lynchburg, Virginia, USA
}

\begin{abstract}
The Asian tiger mosquito (Aedes albopictus) likely arrived in the United States through the importation of used tires. The Asian tiger mosquito is a competent of several arboviruses and with the recent introduction of Zika virus into North America finding methods to control this species is vital. The rapid action of a spray formulation of Bacillus thuringiensis israelensis using Entobac with Aquabac XT is discussed. There was significant reduction of larvae was achieved in less than seven days in the test site whereas the control site showed no significant change. Entobac is currently the only Bti formulation available that controls both adult and larvae mosquitoes. Entobac is also the only Bti formulation that can be utilized in the five principle methods of dispersal; 1) hand tossed 2) backpack spray, 3) truck mounted spray, 4) aerial spray and 5) bait station.
\end{abstract}

\section{Introduction}

The Asian tiger mosquito (Aedes albopictus) first arrived in the United States in the mid1980's, most likely through the importation of used tires (Novak, 1995). Since that time it has become firmly established throughout the southern and eastern United States. Transport of used and shredded tires continue to facilitate the recruitment of this species and storage sites for used tires serve as islands for local populations to spread into local communities (Hawley et al. 1987, Hanson et al. 1996).

Controlling Ae albopictus in tire sites can be problematic for application of pesticides, e.g. restricted openings, stacking of tires, conducive micro-habitat, variability of pesticide efficacy and penetration. Aerial application and truck mounted ULV appear to have only negligible and short term effect in reducing adult $A e$ albopictus populations at tire sites. Baited eugenol pesticide using backpack sprayers and bait stations have been used to effectively reduce adult Aedes populations at tire sites in Florida(Revay et al. 2014). For larval mosquito control, removal of tires and hand tossed granular pesticide application can be effective (GarzaRobledo et al. 2011, Yee et al. 2015). However, when treating stacks of tires, reducing larval populations of mosquitoes can be dangerous;technicians use ladders andexpensive equipmentis often needed to get above stacks of tires, e.g. such as cherry picker equipped vehicles (Novak et al 1990). Faraji and Unlu (2016) stated, "Nonetheless, in the absence of insecticide resistance, when biopesticide or chemical insecticides are applied to container habitats, they are extremely efficacious; however, the main obstacle remains with the logistic challenges in the delivery of these products into ubiquitous, recurring, and inaccessible container habitats." The present study addresses the efficacy of using a baited spray formulation ofBacillus thuringiensis israelensis (Bti) to effectively reduce larval Ae albopictusin tire stacks at a site in Savannah, Georgia, USA.

\section{Methods and Materials}

The Bioagent Transport and Environmental Modeling System TIGER model has been used to model the Transport, Import, Gap, Escalade and Residence and Recruitment of invasive mosquito species and to optimize surveillance sites for vector-borne diseases in several countries (Kollars 2016a). The BioTEMS ${ }^{\text {TM }}$ TIGER model was used to assist in identifying surveillance sites for mosquitoes and vector-borne diseases in Chatham County, GA. Several sites in Savannah, Chatham County, Georgia were identified as high risk areas should local transmission of Zika virus by the Asian tiger mosquito take place. Two tire sites, approximately five miles apartin Savannahwere selected for the study, one control and one test. Each tire site contained over 100 tires in stacks of up to 10 tires.

Entobac $^{\mathrm{TM}}$ formula, with Aquabac ${ }^{\circledR} \mathrm{XT}$ as the source of Bti, is designed to preserve efficacy and to facilitate dispersal of Bti even beyond the point of initial application, by being transported by adult mosquitoes (Yalwala et al. 2016; Kollars et al. 2016b). Entobac ${ }^{\mathrm{TM}}$ can be used as a solid and used in a bait station or can be placed into water and sprayed. Entobac is the first dual use Bti formulation effective against both adult and larval mosquitoes. Entobac is also formulated in such a way as to preserve the efficacy of Bti under harsh conditions even up to six months when used in a bait station.In this study, Entobac ${ }^{\mathrm{TM}}$ was applied by spray to tires at the Test Site using the label rate of 1 pint per acre utilizing a Solo battery powered back pack sprayer. 
Ae albopictus larvae were collected from 15 tires each, at the Test and Control Sites on days 0 and 7 days after Entobac $^{\text {TM }}$ Bti application. Statistica ${ }^{\circledR}$ software was used to analyze the data.

\section{Results and Discussion}

The Entobac ${ }^{\mathrm{TM}}$ Bti formulation was effective in rapidly reducing mosquito populations in tires in Savannah, Georgia. Before application of Entobac ${ }^{\mathrm{TM}}$, there was not a significant difference in the mediannumber of larvae between the Control and Test sites, Mann Whitney U Test, Z=0.394, p=0.685, on Day 0 (Figure 1). By Day 7, after Entobac ${ }^{\mathrm{TM}}$ applicationthere were no live larvae found in the tires at the Test Site although cadavers were found. There was a significant difference between the Test and Control Sites, $\mathrm{Z}=-3.71$, $\mathrm{p}=0.01$ (Figure 2) on Day 7. Entobac ${ }^{\mathrm{TM}}$ has been utilized in bait stations to attract and kill adult mosquito species in several countries, including Aedes, Anopheles, and Culex species. Laboratory trials showed lethal effects of Entobac ${ }^{\mathrm{TM}}$ on larvae at a ratio of 3:1 (larvae to adult),after larvae were exposed to adult Aeaegyptikilled with Entobac ${ }^{\mathrm{TM}}$ (Yalwala et al. 2016).

A number of abiotic and biotic variables can affect the activity of Bti under field conditions. Controlled trials have shown efficacy of Bti up to 6 months (Benjamin et al. 2005). Several studies have shown the efficacy of various Bti in granular formulations in tires and other containers. Under controlled field conditions, mortality levels of at least $70 \%$ were attained for up to 5 weeks for both Vectobac $®$ and Aquabac ( formulations when applied to corn cob granules (Pereira Lima et al. 2005). However, under field conditions residual effectiveness has lasted from as little as 2 days to up to 4 weeks (Batra et al. 2000; Toma et al. 2003). Under similar conditions of being used in tires theefficacy of Vectobac $®$ Bti granules was $90 \%$ at 2 weeks and $50 \%$ at 4 weeks (Kramer, 1990). In open buckets in a tire dump, there was some residual activity after 4 weeks (Farajollahi et al. 2013). To the best of our knowledge, Entobac ${ }^{\mathrm{TM}}$ is the first formulation of Bti that can be used as a solid, liquid or used within a bait station/applicator. In addition to the direct effect of Entobac ${ }^{\mathrm{TM}}$ Bti on adult and larva mosquitoes, this study further provides evidence outside of laboratory trials that adult Aedes species will deliver lethal concentrations of Bti to larvae, further reducing mosquito populations.

Studies of tire sites are important in understanding vector dynamics; tire sites can be abundant and found close to human populations, adding to the habitat range for vector mosquitoes (Yee, 2008). BioTEMS has been used to model biological weapons,infectious diseases and vectors to assist in risk and vulnerability assessments for U.S. presidential security, military bases and civilian populations in several countries. Pest control operations often utilize a one mile area for area wide control and as little as a 150 meter radius for space spraying to control vector species. The reaction to Zika invading Miami, Florida, USA has shown that these recommended minimum areas are not sufficient to prevent an outbreak. The BioTEMS TIGER model indicates an initial surveillance and control area for Aedes vectors of Zika and other arboviruses of 2.4 miles. An area of 9.5 miles was indicated for supplemental surveillance to increase detection in the event of Gap infiltration by Aedes species.

There are five main mechanisms of applying pesticides to control mosquito vectors; 1) hand/backpack sprayers, 2) bait stations, 3) hand tossing granules, 4) truck-mounted sprayers, and 5) aerial application. Identifying both the area at risk and optimizing surveillance sites in probable hot zonesis essential for preventing invasion of Zika virus, reducing the impact of an outbreak, preventing recruitment of infected conspecificsfrom other geographic regions.Aerial and truck mounted application of adulticides for control of Ae albopictus and Ae aegyptican contribute to control but their short term effectiveness must be incorporated into the integrated mosquito management plan. For example, truck mounted spraying of Bti using a cold thermal- fogger was effective in penetrating into the surrounding habitat of bushes and reducing Ae albopictus larvae (Williams et al. 2014).In addition to the frequency and cost of area-wide larvicide application, most operations use formulations intended for other uses andare only conducted with equipmentavailable on hand (Faraji and Unlu, 2016). A critical step forward in the control of Aedes has been made with the application of Entobac ${ }^{\mathrm{TM}}$ using Aquabac ${ }^{\circledR}$ $\mathrm{XT}$, the advantages being; 1) dual use against adult and larval mosquitoes, 2) Bti isvirtually non-toxic according to the U.S. Environmental Protection Agency, 3)multiplication effect by delivery and transmission of Btiby adults to larvae, 4) environmental stability, and 5) Entobac ${ }^{\mathrm{TM}}$ can be applied using any of the five pesticide application technologies.

\section{Acknowledgements}

The author is the owner of the Entobac ${ }^{\mathrm{TM}}$ formulation. Aquabac ${ }^{\circledR}$ and Vectobac ${ }^{\circledR}$ were purchased from distributors. The views and conclusions expressed do not reflect the official policy or position of the US Army, Department of Defense, or the U.S. Government. 
Figure 1.There was no significant difference between the median number of Aedes albopictus larvae at the Test Site (TS) versusControl Site $(C)$ on Day $0(p \geq 0.05)$.

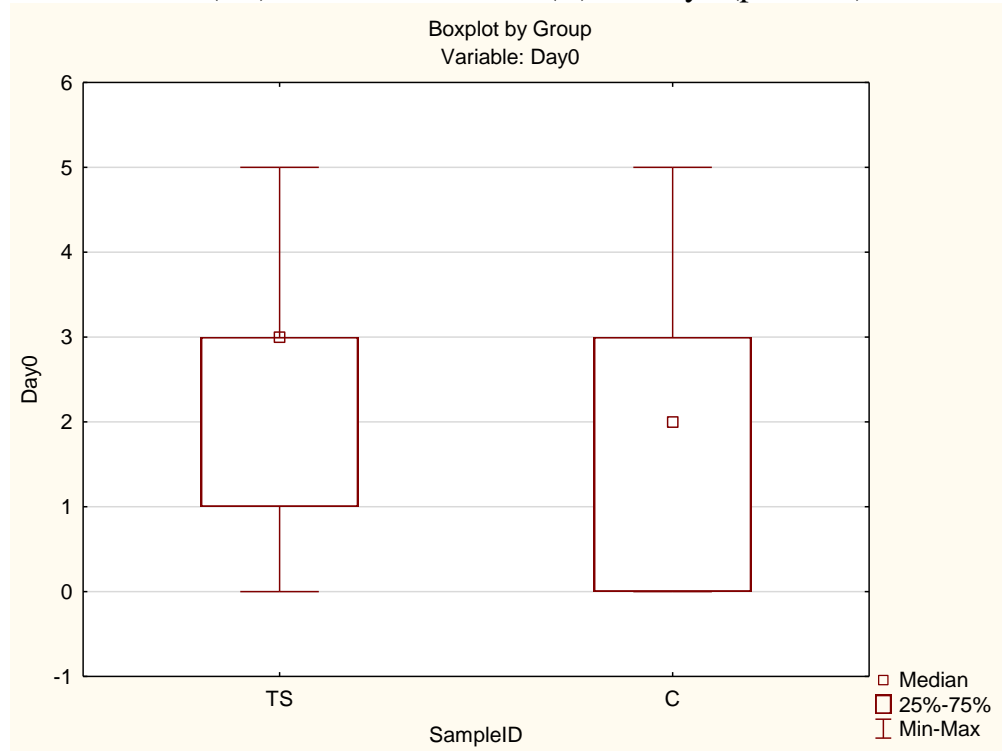

Figure 2.There was a significant difference between the mediannumberof Aedes albopictus larvae at the Test Site (TS) versusControl Site $(\mathrm{C})$ on Day 7 ( $\mathrm{p} \leq 0.05)$.

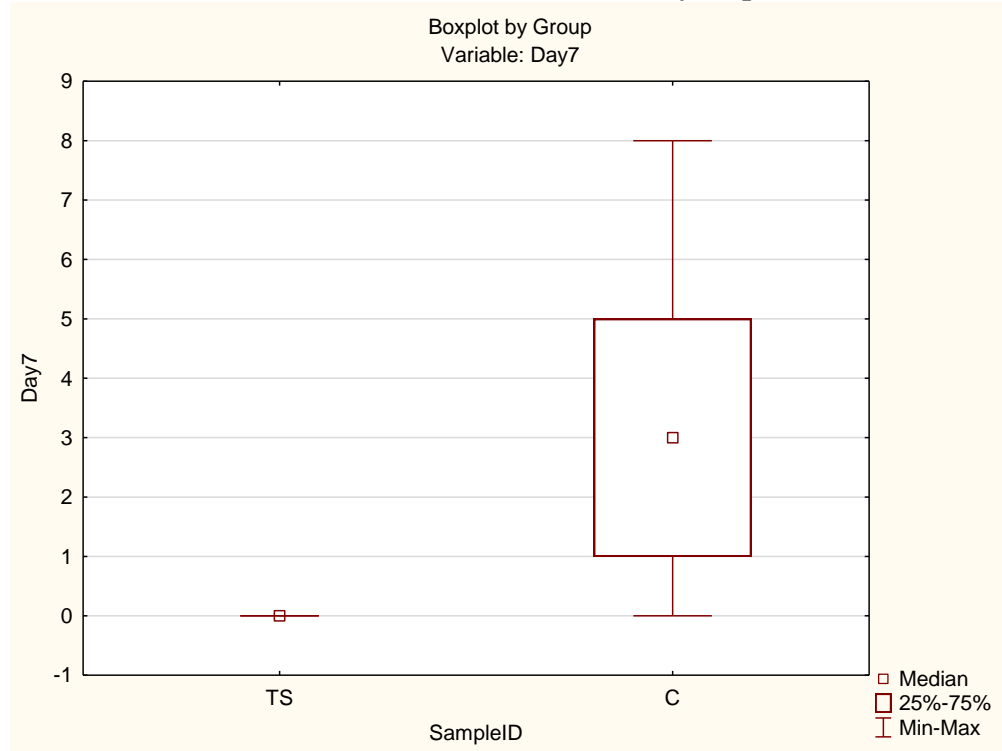

\section{References}

[1]. Batra CP, PK Mittal, T Adak (2000). Control of Aedes aegypti breeding in desert coolers andtires by use of Bacillus thuringiensis var. israelensis formulation. Journal of the American Mosquito Control Association 16:321-3.

[2]. Benjamin S, A Rath, YF Chiang, LH Lim (2005). Efficacy of Bacillus thuringiensis israelensistablet formulation Vectobac DT for control of dengue mosquito vectors in potable containers. Southeast Asian Journal of Tropical Medicine and Public Health 36: 879892.

[3]. Faraji, A and I Unlu (2016). The eye of the tiger, the thrill of the fight: effective larvalandadult control measures against the Asian tigermosquito, Aedes albopictus (Diptera: Culicidae), inNorth America. Journal of Medical Entomology, 53: 1029-1047.

[4]. Farajollahi, A, GM Williams, GC Condon, B Kesavaraju, and I Unlu (2013). Assessment of adirect application of two Bacillus thuringiensis israelensis formulations for immediate and residual control of Aedes albopictus. Journal of the American Mosquito Control Association 29: 385-388.

[5]. Garza-Robledo, AA, JF Martinex-Perales, VA Rodriguez-Castro and H Quiroz-Martinez (2011).Effectiveness of spinosad and temephos for the control of mosquito larvae at a tire dump in Allende, Nuevo leon, Mexico. Journal of the American Mosquito Control Association 27:404-407.

[6]. Hanson SM, RL Lampman, RJ Novak, and PM Purseglove (1996). Aedes albopictus and Ae.triseriatus eggs survive waste tire processing. J Am Mosq Control Association 12:728-729.

[7]. Harwood JF, MFarooq, BTTurnwall, and AGRichardson (2015). Evaluating Liquid andGranular Bacillus thuringiensis var. israelensis Broadcast Applications for Controlling Vectors of Dengue and Chikungunya Viruses in Artificial Containers and Tree Holes.Journal of Medical Entomology 52:663-671. 
[8]. Hawley WA, P Reiter, RS Copeland, CB Pumpuni, GB CraigJr. (1987). Aedes albopictus inNorth America: probable introduction in used tires from northern Asia. Science 236:1114-1116.

[9]. Kollars, TM Jr., P Kollars, and B Hulsey (2016a) Preventive Medicine: Reducing the risk tomarine ports from invasive mosquito species, Zika, dengue, Chikungunya viruses and filariasis. International Journal of Medicine (in press).

[10]. Kollars TM, S Hatfill, EL Kollars, and K Neidhardt (2016b). Controlling mosquito populations and reducing the risk of mosquitoborne diseases in a beach resort of Punta Cana using eco-friendly technologies.IOSR Journal of Pharmacy and Biological Sciences 11: $72-75$.

[11]. Kramer, VL (1990). Efficacy and persistence of Bacillus sphaericus, Bacillus thuringiensis var.israelensis, and methoprene against Culisetaincidens (Diptera: Culicidae) in tires.Journal of Economic Entomology 83: 1280-1285.

[12]. Novak, RJ (1995). A North American model to contain the spread of Aedes albopictus throughtirelegislation. Parassitologia 37:12939.

[13]. Novak, RJ, BA Steinly, DW Webb, L Haramis, J Clarke Jr., B Farmer, and R Cieslik (1990).Penetration rate of two pesticide carriers at a large used-tire storage facility in Chicago, Illinois. Journal American Mosquito Control Association 6: 188-196.

[14]. Pereira Lima, JB, N Vieira de Melo, D Valle (2005). Residual effect of two Bacillusthuringiensis var. israelensis products assayed against Aedes aegypti (Diptera: Culicidae) in laboratory and outdoors at Rio de Janeiro, Brazil. Journal of the São Paulo Institute of Tropical Medicine 47:125-130.

[15]. Revay, EE, GC Mueller, WA Qualls, DL Kline, DP Naranjo, KLArheart, VD Kravchenko, ZYefremova, AHausmann, and JC Beier (2014). Control of Aedes albopictus with attractive toxic sugar baits (ATSB)and potential impact on non-target organisms in St. Augustine. FloridaParasitology Research 113: 73-79.

[16]. Toma L, Severini F, Bella A, Romi R (2003). A semifield evaluation of Vectobac DT (ABG-6499), a new formulation of Bacillus thuringiensis israelensis for control of Aedes albopictus. Journal of the American Mosquito Control Association. 19:424-429.

[17]. Williams, GM, A Faraji, I Unlu, SP Healy, M Farooq, R Gaugler, G Hamilton, DM Fonseca(2014). Area-wide ground applications of Bacillus thuringiensis var. israelensis for the control of Aedes albopictus in residential neighborhoods: from optimization to operation. PLoS One 9.10 : e110035

[18]. Yalwala, S, JW Kollars, G Kasembeli, C Barasa, C Senessie, PG Kollars, TM Kollars Jr (2016).Preliminary report on the reduction of adult mosquitoes in housing compounds in western Kenya using the ProVector Flower and Entobac Bait Pads containing Bacillus thuringiensis israelensiswith honey bait. Journal of Medical Entomology 53: 1242-1244.

[19]. Yee, DA (2008). Tires as habitats for mosquitoes: A review of studies within the eastern UnitedStates. Journal of Medical Entomology 45: 581-593.

[20]. Yee DA, AA Abuzeineh, NF Ezeakacha, SS Schelble, WC Glasgow and SD Flanagan (2015). Mosquito Larvae in Tires from Mississippi, United States: The efficacy of abiotic and biotic parameters in predicting spatial and temporal patterns of mosquito populations andcommunities. Journal Medical Entomology 51: 394-407. 